\title{
Survival probability for chaotic particles in a set of area preserving maps
}

\author{
Juliano A. de Oliveira ${ }^{1, a}$, Diogo R. da Costa $^{2}$, and Edson D. Leonel ${ }^{2,3}$ \\ 1 UNESP, University Estadual Paulista, Câmpus de São João da Boa Vista, Av. \\ Professora Isette Corrêa Fontão, 505, Jardim Santa Rita das Areias, 13876-750, São João \\ da Boa Vista, SP, Brazil \\ 2 Departamento de Física, UNESP, University Estadual Paulista, Av.24A, 1515, Bela \\ Vista, 13506-900, Rio Claro, SP, Brazil \\ 3 Abdus Salam International Center for Theoretical Physics, Strada Costiera 11, 34151 \\ Trieste, Italy
}

Received 21 December 2015 / Received in final form 25 February 2016 Published online 22 November 2016

\begin{abstract}
We found critical exponents for the dynamics of an ensemble of particles described by a family of Hamiltonian mappings by using the formalism of escape rates. The mappings are described by a canonical pair of variables, say action $J$ and angle $\theta$ and the corresponding phase spaces show a large chaotic sea surrounding periodic islands and limited by a set of invariant spanning curves. When a hole is introduced in the dynamical variable action, the histogram for the frequency of escape of particles grows rapidly until reaches a maximum and then decreases towards zero for long enough time. The survival probability of the particles as a function of time is measured and statistical investigations show it is scaling invariant with respect to $\gamma$ and time for chaotic orbits along the phase space.
\end{abstract}

\section{Introduction}

In the last years, different problems were considered in order to characterize the chaotic dynamics described by two-dimensional nonlinear and area-preserving mappings. Applications can be seen in study of channel flows, waveguide, transport properties, Fermi acceleration and also for the study of magnetic field lines in toroidal plasma devices with reversed shear (like tokamaks) [1-6].

Generally, the mappings have more than a control parameter. One of them control the intensity of the non-linearity hence controlling also a transition from integrability to non integrability. In the majority of the cases, the phase spaces of the mappings are mixed. They are composed by a chaotic sea that eventually surrounds periodic islands and is limited by a set of invariant spanning curves. The size of the chaotic sea is strongly influenced by the control parameters since they play the rule of controlling the intensity of the nonlinearity.

\footnotetext{
a e-mail: julianoantonio@sjbv.unesp.br
} 
Recently a detailed study of statistical properties such as deviation of average along the chaotic sea for a class of two-dimensional Hamiltonian mappings were made to allow connections with other models [7,8]. Statistical properties are also close related to the statistics of escape from a region containing a hole [9]. These properties provide useful information for the control of chaotic systems [10]. Escape properties measured as a function of a varying hole provide a further sensitive and non-destructive probe of the dynamics [11]. Recently this approach has also been successfully applied in a Hamiltonian system to show that the diffusion coefficient is scaling invariant in the fully chaotic region [12].

In this paper, we investigate some escape properties in a special class of two dimensional nonlinear and area-preserving mapping. We then study the escape properties along the chaotic sea and therefore find critical exponents that allow us to define and compare classes of universality. Such critical exponents are confirmed in terms of scaling hypotheses. The critical exponents are obtained for several values of the control parameters.

This paper is organized as follows: in Sect. 2 we define the model and obtain our numerical results. In Sect. 3 we present the final discussions.

\section{The model and numerical results}

We consider in this paper a mapping that can be obtained from a perturbed Hamiltonian. In generalized coordinates the controlling Hamiltonian is given by [13],

$$
H\left(J_{1}, J_{2}, \theta_{1}, \theta_{2}\right)=H_{0}\left(J_{1}, J_{2}\right)+\epsilon H_{1}\left(J_{1}, J_{2}, \theta_{1}, \theta_{2}\right),
$$

where the variables $J_{i}$ and $\theta_{i}$ with $i=1,2$ are respectively the action and angle. $H_{0}$ represents the integrable part while $H_{1}$ is the non integrable part of the Hamiltonian. One sees that the control parameter $\epsilon$, which defines the magnitude of coupling controls a transition from integrability to non integrability. To characterize the dynamics in terms of a mapping, we consider a Poincaré section defined by the plane $J_{1} \times \theta_{1}$ and assume $\theta_{2}$ as constant $(\bmod 2 \pi)$. A generic two dimensional map which qualitatively describes the behavior of (1) is

$$
T:\left\{\begin{array}{l}
J_{n+1}=J_{n}+\epsilon \tilde{h}\left(\theta_{n}, J_{n+1}\right) \\
\theta_{n+1}=\left[\theta_{n}+F\left(J_{n+1}\right)+\epsilon p\left(\theta_{n}, J_{n+1}\right)\right] \bmod (2 \pi)
\end{array}\right.
$$

where $\tilde{h}, F$ and $p$ are assumed to be nonlinear functions of their variables and the index $n$ denotes the $n$th iteration of the mapping. Since the map, in Eq. (2) should be area preserving, the expressions for $\tilde{h}\left(\theta_{n}, J_{n+1}\right)$ and $p\left(\theta_{n}, J_{n+1}\right)$ must satisfy the following condition

$$
\frac{\partial p\left(\theta_{n}, J_{n+1}\right)}{\partial \theta_{n}}+\frac{\partial \tilde{h}\left(\theta_{n}, J_{n+1}\right)}{\partial J_{n+1}}=0
$$

For many mappings considered in the literature, the function $\tilde{h}\left(\theta_{n}\right)=\sin \left(\theta_{n}\right)$ and we have liberty to chose $p$ and $F$. Without losing generality, for a wide class of systems the function $p$ is considered $p\left(\theta_{n}, J_{n+1}\right)=$ constant which we will consider it as fixed $p\left(\theta_{n}, J_{n+1}\right)=0$ from now on and hence $F$ is varied. The systems within the scope of the general two dimensional map include, the logistic twist mapping [14], the Taylor-Chirikov map [15], Fermi-Ulam accelerator model [16,17], Fermi-Pustylnikov accelerator [18] or bouncer model and Hybrid-Fermi-Ulam-bouncer model [19,20]. 


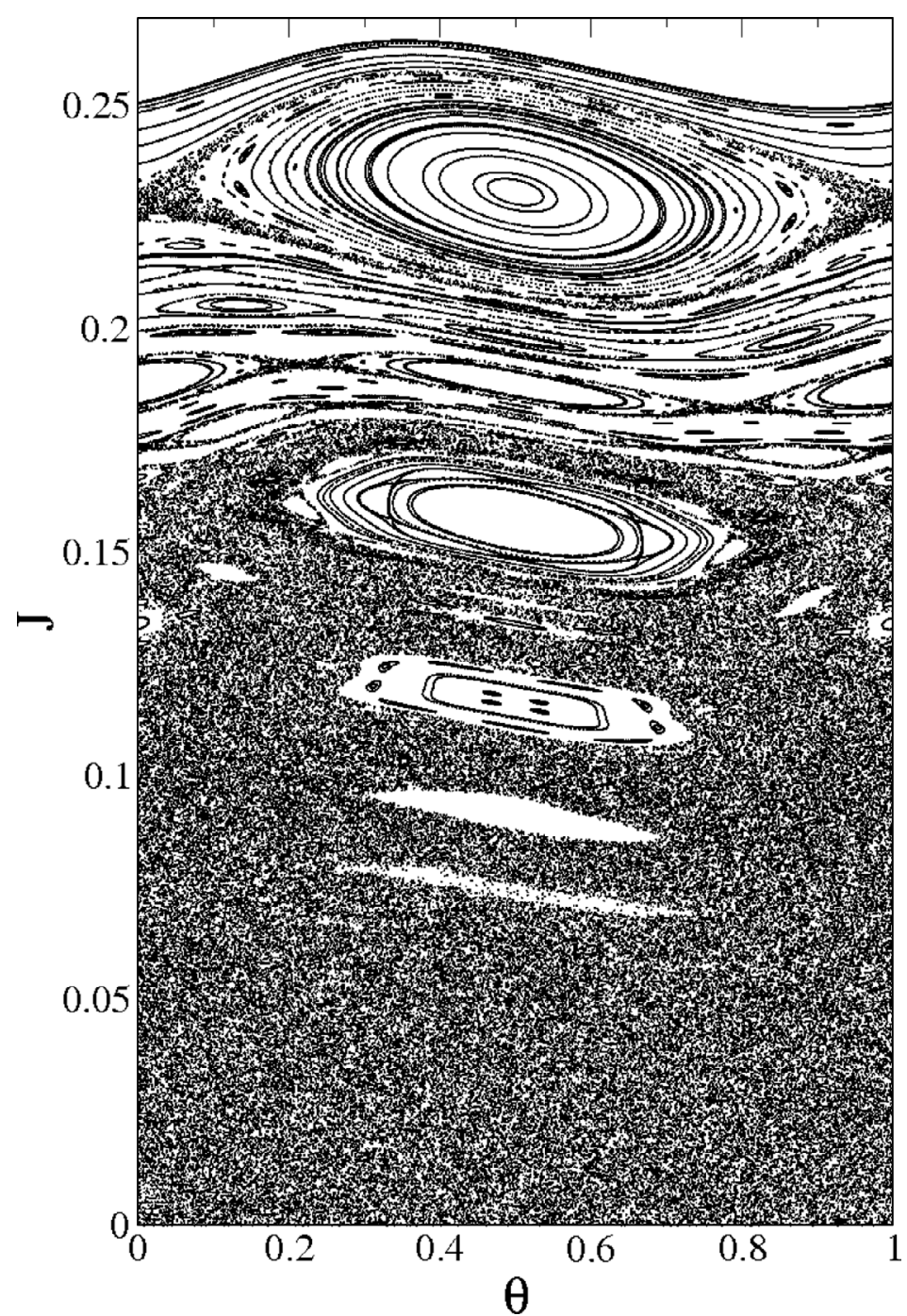

Fig. 1. Phase space for mapping (2) using $\epsilon=10^{-2}$ and $\gamma=3 / 4$.

In this work, our main goal is to investigate the dynamical properties for an ensemble of particles considering the following expression

$$
F(J)=|J|^{-\gamma},
$$

where $0<\gamma \leq 1$ is a control parameter. Incorporating the functions $F\left(J_{n+1}\right)=$ $1 /\left|J_{n+1}\right|^{\gamma}, p=0$ and $\tilde{h}=\sin \left(\theta_{n}\right)$, the map has assumes the following form

$$
T:\left\{\begin{array}{l}
J_{n+1}=J_{n}+\epsilon \sin \left(\theta_{n}\right) \\
\theta_{n+1}=\left[\theta_{n}+\frac{1}{\left|J_{n+1}\right|^{\gamma}}\right] \bmod (2 \pi)
\end{array} .\right.
$$

In this case, a detailed study of the average properties for the action as a function of the control parameters as well as the time was recently discussed in $[7,8]$. The phase space generated from mapping (5) is shown in Fig. 1. One sees that the phase space 
(a)

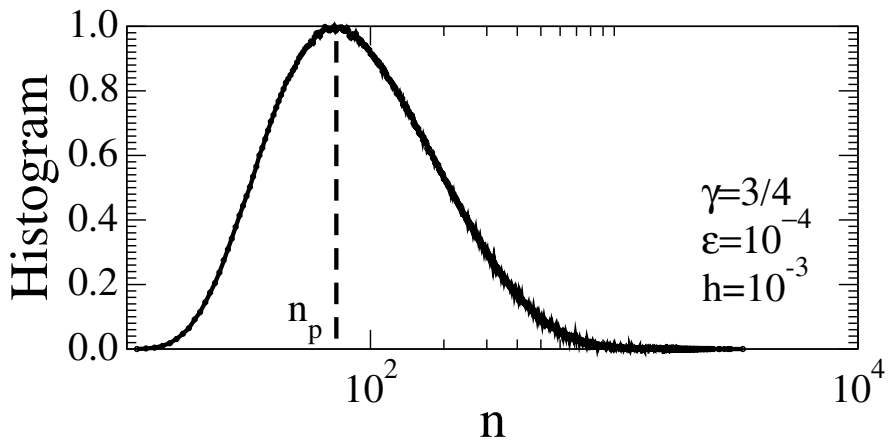

(b)

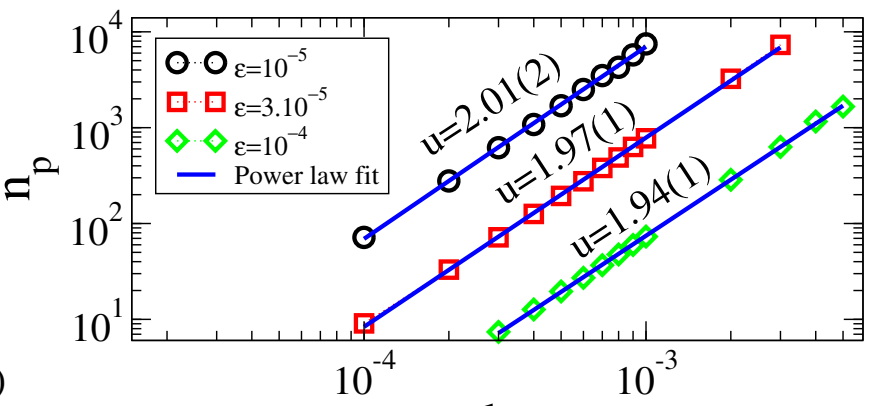

$\mathrm{h}$

(c)

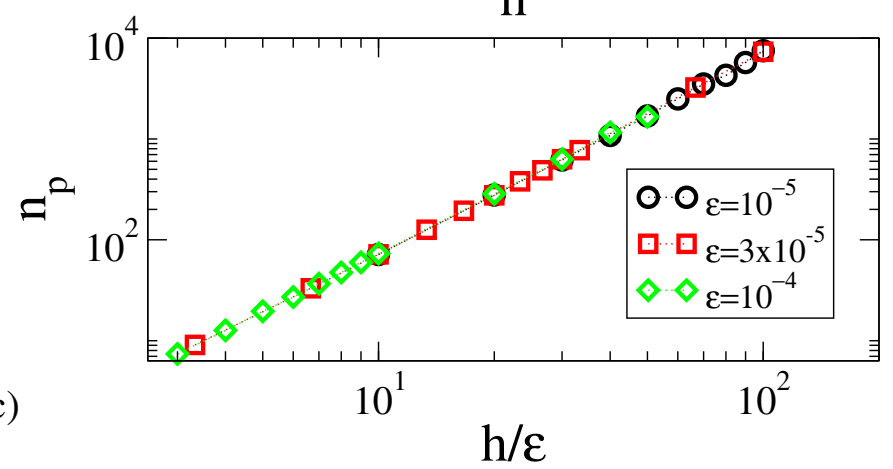

Fig. 2. For $\gamma=3 / 4$ we have: (a) A plot of the histogram as a function of $n_{p}$, where $n_{p}$ was highlighted; (b) $n_{p} \times h$ for different values of $\epsilon$, namely: $\epsilon=10^{-5}, \epsilon=3.10^{-5}$ and $\epsilon=10^{-4}$; (c) $n_{p} \times h / \epsilon$.

is mixed and contains a set of periodic islands surrounded by a large chaotic sea that is limited by a set of invariant spanning curves.

We consider a set of particles with initial conditions in a region of small $J$ (we take $J_{0}=0.01$ in our simulations). For this set of particles, if one of them has $J \geq h-$ which is a typical position in the phase space - we assume the particle reached a hole and then through it, the particle escapes. After escaping, the particle is eliminated from the simulation, the number of iterations spent until the escape is registered in a vector and a new initial condition, with a different phase and same initial $J_{0}$ is started. The procedure is repeated until the ensemble is exhausted. Figure 2(a) shows a histogram (or frequency) for the number of particles that escaped for an iteration $n$. We notice the histogram starts to growth with for short $n$ and, eventually, reaches a maximum value for a characteristic $n_{p}$, while after the peak it starts to decrease to zero asymptotically. 


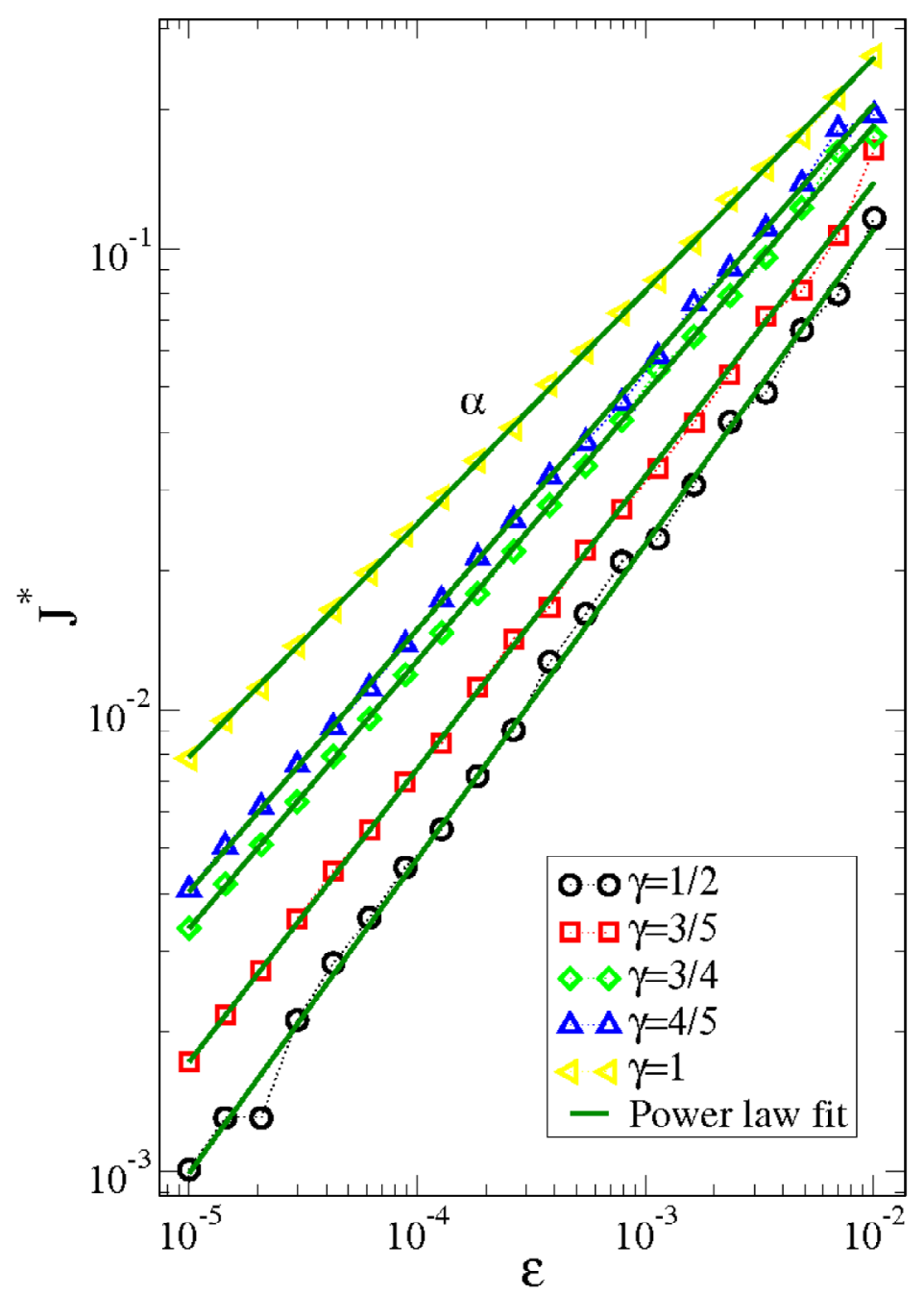

Fig. 3. A closest approximation for the position of the lowest invariant spanning curve $J^{*}$ as a function of the parameter $\epsilon$ for five different values of $\gamma$, namely: $\gamma=1 / 2, \gamma=3 / 5$, $\gamma=3 / 4, \gamma=4 / 5$ and $\gamma=1$.

In Fig. 2(b) we show $n_{p}$ as a function of $h$ for three different values of $\epsilon$. After a scaling transformation $h \rightarrow h / \epsilon$, all curves shown in 2(b) are overlapped onto each other as shown in Fig. 2(c). Therefore, we can write that

$$
n_{p} \propto\left(\frac{h}{\epsilon}\right)^{u},
$$

where $u \approx 2$ is the slope for $n_{p}$ as a function of $h$.

In Fig. 3 we show an approximated position for the first invariant spanning curve for different values of $\gamma$. To obtain such a position, we divided the $\theta$ axis in 1,000 peaces uniformly spaced along the range $[0,1]$ and evolved a single orbit for over $10^{9}$ iterations. For each window in $\theta$, we keep the corresponding maximum value for a chaotic orbit. When all peaces are joint, the resulting set of points furnishes a closest approximation for the position of the first invariant spanning curve $J^{*}$. The plots 


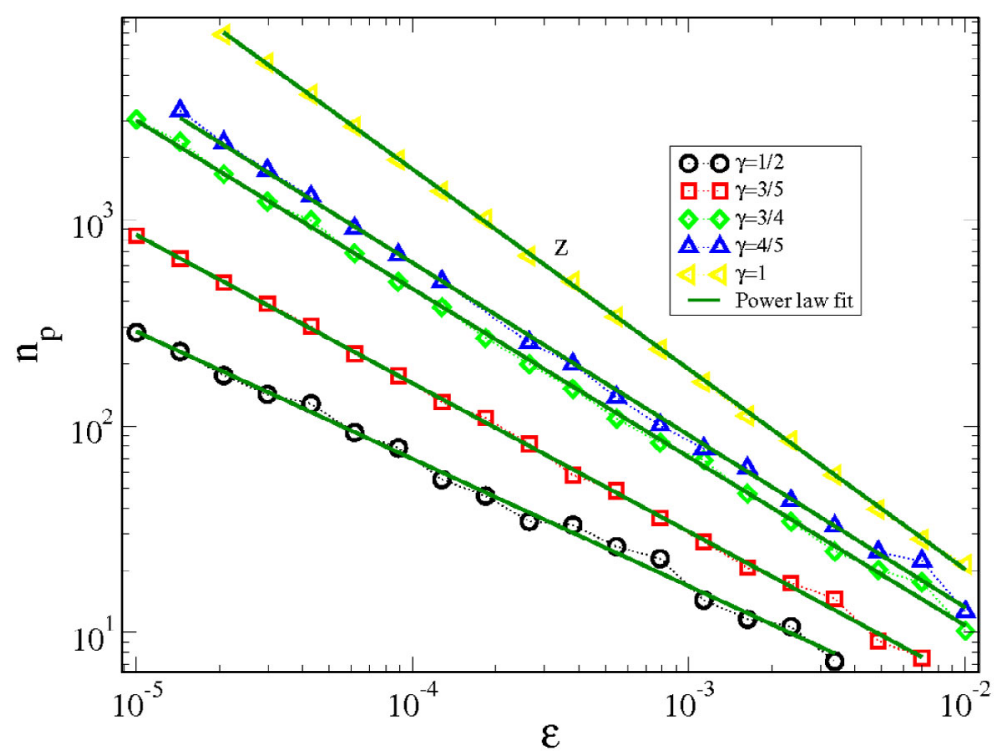

Fig. 4. Plot of $n_{p} v s . \epsilon$ for $\gamma=3 / 4$.

Table 1. Critical exponents obtained numerically for $\epsilon \in\left[10^{-5}, 10^{-2}\right]$.

\begin{tabular}{ccc}
\hline$\gamma$ & $\alpha$ & $z$ \\
\hline $1 / 2$ & $0.674(5)$ & $-0.630(4)$ \\
$3 / 5$ & $0.635(5)$ & $-0.718(6)$ \\
$3 / 4$ & $0.580(3)$ & $-0.814(7)$ \\
$4 / 5$ & $0.569(3)$ & $-0.834(8)$ \\
1 & $0.505(1)$ & $-0.968(4)$ \\
\hline
\end{tabular}

are constructed by the corresponding lowest value of $J^{*}$ along the invariant spanning curve. Figure 3 shows $J^{*}$ as a function of $\epsilon$, and after a power law fit we obtained the critical exponent $\alpha$ for different values of $\gamma$. We can conclude that

$$
J^{*} \propto \epsilon^{\alpha}
$$

Figure 4 shows $n_{p}$ as a function of $\epsilon$ for different values of $\gamma$. We considered

$$
h=c_{p} J^{*},
$$

where $c_{p}$ is chosen to be $20 \%$ of the higher of $J^{*}$. A power law fitting gives the critical exponent $z$. We can see in Table 1 a set of critical exponents $\alpha$ and $z$ for different values of $\gamma$. Using a scaling hypothesis we can write

$$
n_{p} \propto \epsilon^{z}
$$

Comparing Eq. (6) and Eq. (8) we can obtain that

$$
n_{p} \propto\left(\frac{c_{p} J^{*}}{\epsilon}\right)^{u}
$$


and using Eq. (9) we have

$$
\epsilon^{z+u} \propto\left(J^{*}\right)^{u}
$$

Now using this last expression and combining with Eq. (7) we obtained

$$
\epsilon^{z+u} \propto \epsilon^{\alpha u}
$$

We conclude that

$$
z=\alpha u-u \text {. }
$$

There is an interesting interpretation for $u$. When comparing the results with those used in the scaling hypothesis we obtained $u=1 / \beta$, where $\beta=0.5$ for many problems with normal diffusion. In our simulations, we obtained $u \approx 2$, therefore the equation for $z$ can be written as

$$
z=\frac{\alpha}{\beta}-\frac{1}{\beta}
$$

We show in Fig. 5(a) the histogram for the frequency of escape as a function of $n$ using different values of $h$ and $\epsilon$. One can see that depending on the parameters, the curves have different values of $n_{p}$. If a rescale $n \rightarrow n /\left(c_{p}^{u} \epsilon^{z}\right)$ is applied in the horizontal axis, all curves overlap each other onto a single and universal curve as shown in Fig. 5(b), hence confirming the scaling invariance of the histogram. The vertical axis was chosen such that the maximum value was set as one.

Let us now to discuss the behavior of the survival probability, which is defined as

$$
P=\frac{1}{N} \sum_{j=1}^{N} N_{\text {surv }}(n),
$$

where the summation is taken along the ensemble of $N$ different initial conditions and $N_{\text {surv }}(n)$ is the number of initial conditions that do not escape through the hole until a time $n$. When Eq. (15) is evaluated in a fully chaotic dynamics its behavior is an exponential [11] while for a mixed phase space where periodic orbits exist, the exponential decay may turn into a power law [9] or other laws like stretched exponentials.

The behavior of $P$ vs. $n$ is shown in Fig. 6(a). We see that the typical behavior is an exponential decay $P \propto \exp (\nu n)$. The slope of the decay is plotted as function of the position of the hole, as shown in Fig. 6(b). Figure 6(c) shows an overlap of all curves shown in Fig. 6(b) after the transformation $n \rightarrow h / \epsilon$, hence confirming a scaling invariance of the survival probability as a function of $n$.

For large enough $n$ the diffusion is no longer Brownian. The stickiness surrounding the elliptic islands leads to the power law decays. Interestingly, even in the intermediate time regime, where decay is approximately exponential, there is a subtle deviation from a normal diffusive law: The exponents in Fig. 6(b) are approximately -2 .

In Table 2 we show the critical exponent $\left(z^{\prime}\right)$ obtained from the curves shown in Fig. 7. We can observe that

$$
z^{\prime} \approx-z
$$

where the values of $z$ are shown in Table 1 .

Extensions of the formalism made in this paper can be made for different models. As for example, for the case of $\gamma=1$ and considering the transformations $J \rightarrow V$ and $\theta \rightarrow \phi$, one can recover the so called Fermi-Ulam accelerator model [21]. For the 

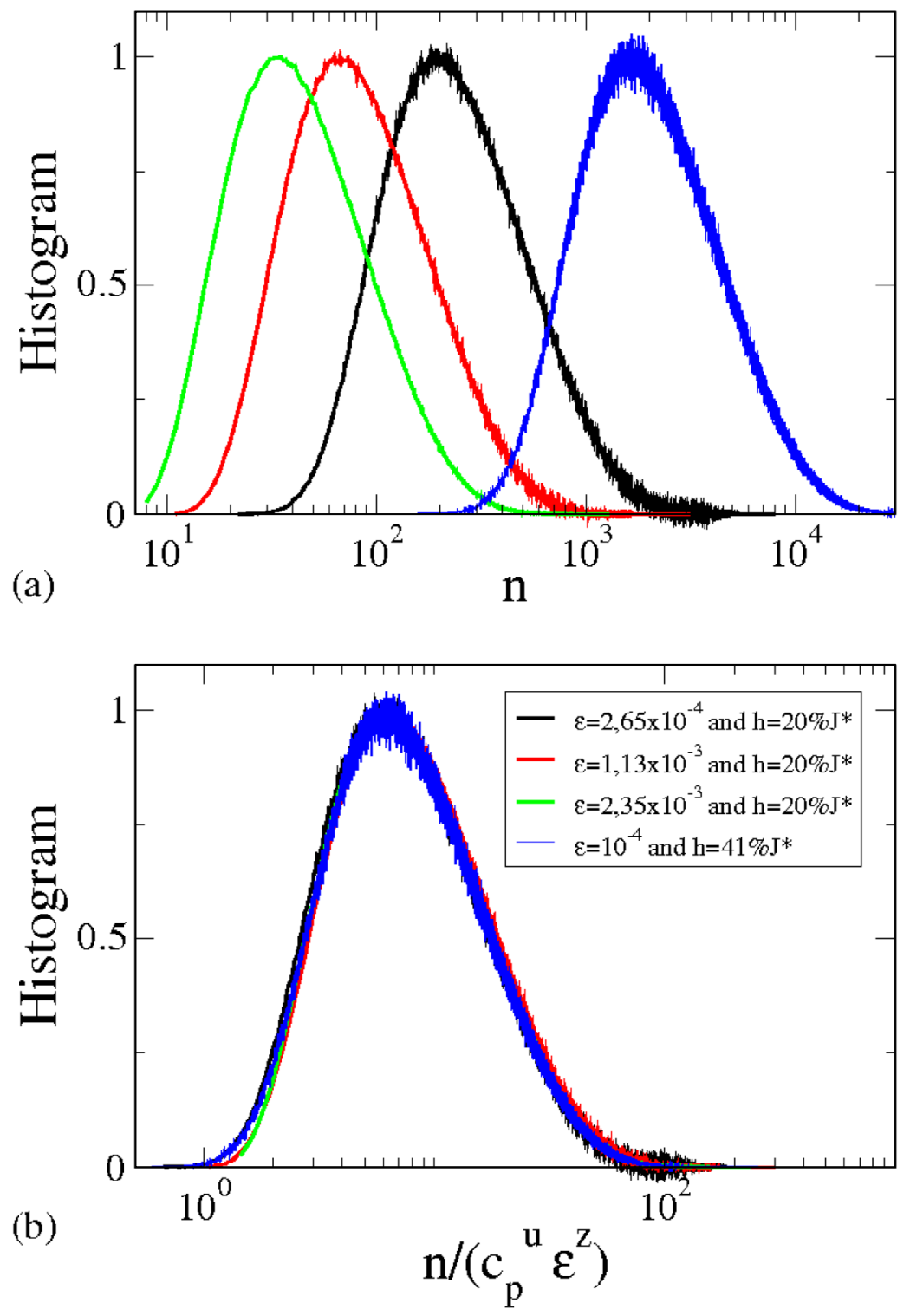

Fig. 5. (a) Plot of the histogram for the frequency of escape as a function of $n$ for four different values of $\epsilon$ and $h$, as labeled in the figure; (b) after rescaling the horizontal axis, all curves overlap onto each other in a single and universal plot.

Table 2. Critical exponent $z^{\prime}$ for different values of $\gamma$.

\begin{tabular}{cl}
\hline$\gamma$ & \multicolumn{1}{c}{$z^{\prime}$} \\
\hline $1 / 2$ & $0.62(1)$ \\
$3 / 5$ & $0.720(5)$ \\
$3 / 4$ & $0.818(7)$ \\
$4 / 5$ & $0.839(8)$ \\
1 & $0.973(4)$ \\
\hline
\end{tabular}



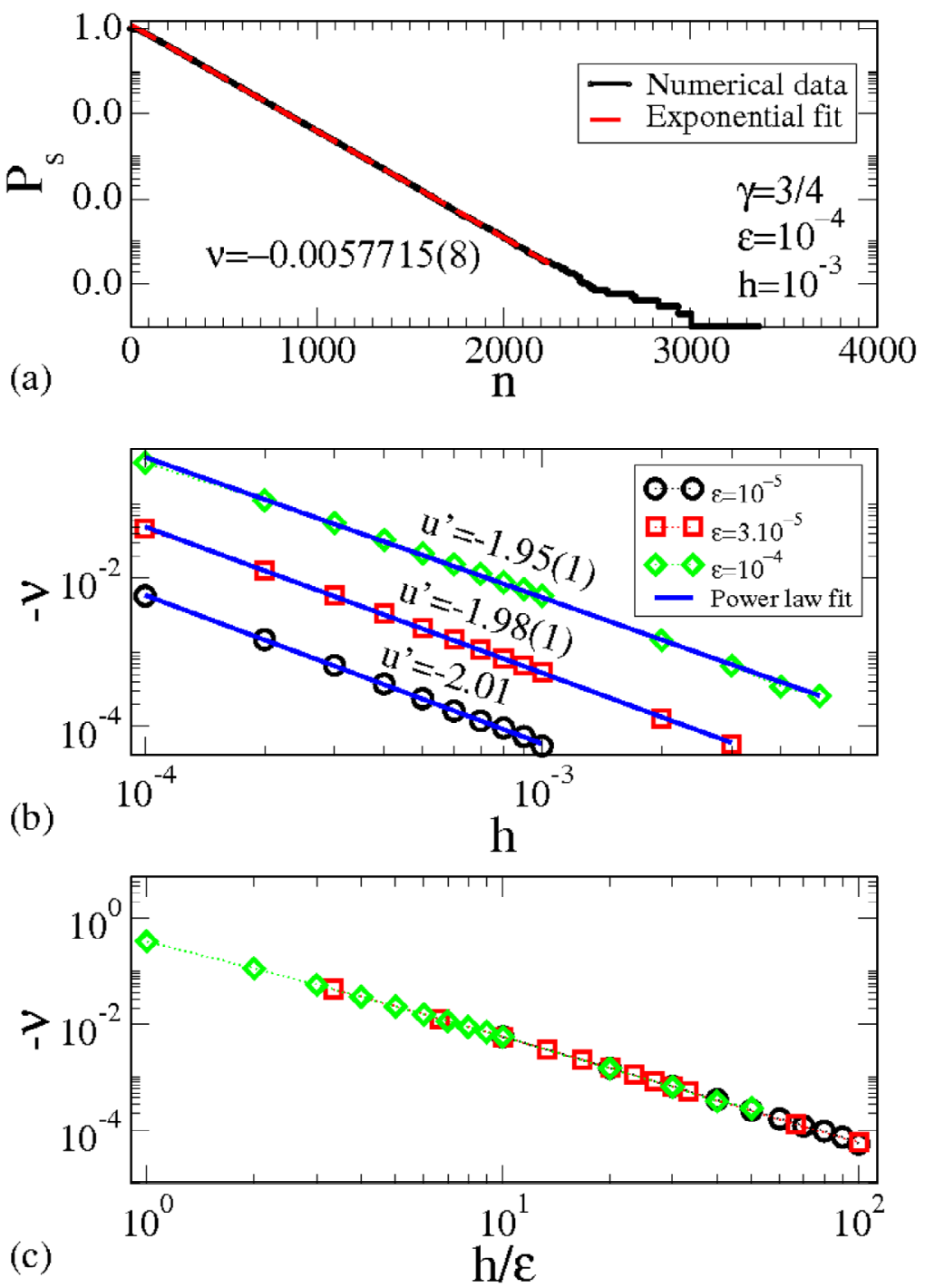

Fig. 6. (a) Plot of $P_{s}$ vs. $n$ confirming exponential behavior for small $n$. The slope obtained is $\nu=-0.0057715(8)$; (b) plot of $\nu$ vs. $h$ for three different values of $\epsilon$ namely: $\epsilon=10^{-5}$, $\epsilon=3 \cdot 10^{-5}$ and $\epsilon=10^{-4}$; (c) overlap of the curves shown in (b) after the transformation $h \rightarrow h / \epsilon$.

case where $J \rightarrow \gamma$ and $\theta \rightarrow X$ where $\gamma$ in this transformation represents angular coordinate instead of control parameter (as is the case for this paper), one can have the periodically corrugate waveguide [22]. In these two cases, the critical exponents obtained were $\alpha=0.5$ and $z=-1$ which is very well predicted in Table 1 . On the other hand, for the case of $\gamma=1 / 2$, one can describe the dynamics of a classical particle confined inside an infinitely deep box of potential containing a periodically moving square well $[23,24]$ or time varying barrier [25]. For these cases, the critical exponent is $\alpha=0.674(5)$ and $-0.630(4)$, as shown in Table 1. 


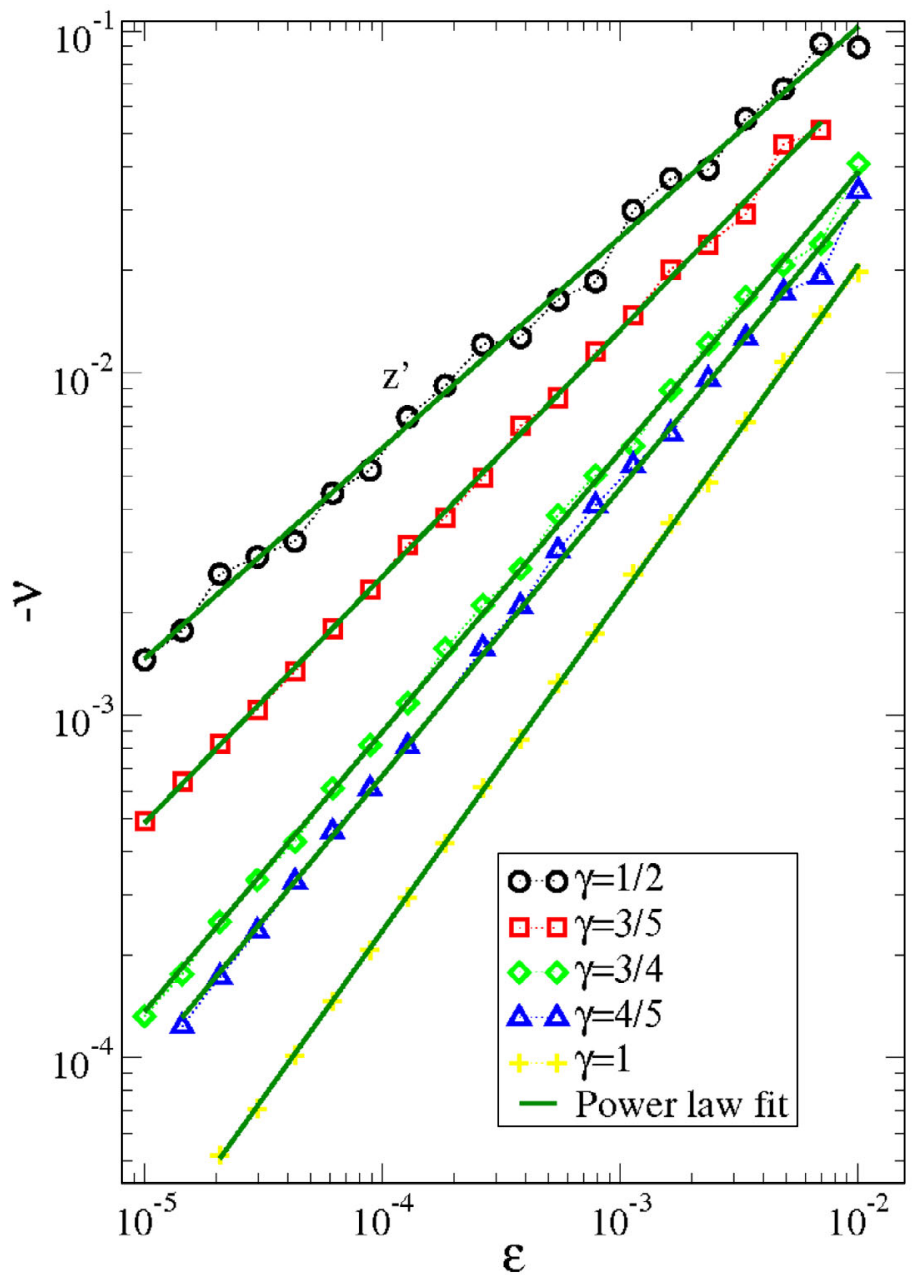

Fig. 7. Plot of $-\nu \times \epsilon$ for different values of $\gamma$ (as labeled in the figure).

\section{Conclusion}

In this paper we have used some escape properties to investigate the behavior of the survival probability for a set particles moving chaotically in a phase space of an area preserving map. Critical exponents were obtained numerically and confirmed using scaling hypothesis for several different values of $\gamma$, denoting then different models.

JAO thanks PROPe/UNESP, CNPq (311105/2015-7) and FAPESP(2014/18672-8) by financial support. DRC acknowledges Brazilian agency FAPESP (2013/22764-2) and EDL thanks to CNPq (303707/2015-1), FUNDUNESP and FAPESP (2012/23688-5) (Brazilian agencies).

\section{References}

1. G.A. Luna-Acosta, J.A. Méndez-Bermudéz, P. Seba, K.N. Pichugin, Phys. Rev. E 65, $046605(2002)$

2. G.M. Zaslavsky, Phys. Rep. 371, 461 (2002)

3. D.G. Ladeira, J.K.L. da Silva, J. Phys. A: Math. Theor. 40, 11467 (2007) 
4. F.R.N. Kock, F. Lenz, C. Petri, F. K. Diakonos, P. Schmelcher, Phys. Rev. E 78, 056204 (2008)

5. J.E. Howard, A.J. Lichtenberg, M.A. Lieberman, R.H. Cohen, Physica D 20, 259 (1986)

6. G.A. Luna-Acosta, K. Na, L.E. Reichl, Phys. Rev. E 53, 3271 (1996)

7. J.A. de Oliveira, R.A. Bizão, E.D. Leonel, Phys. Rev. E 81, 046212 (2010)

8. E.D. Leonel, J. Penalva, R.N.M. Teixeira, R.N. Costa Filho, M.R. Silva, J.A. de Oliveira, Phys. Lett. A 379, 1808 (2015)

9. E.G. Altmann, T. Tél, Phys. Rev. E 79, 016204 (2009)

10. H. Buljan, V. Paar, Phys. Rev. E 63, 066205 (2001)

11. L.A. Bunimovich, C.P. Dettmann, EPL 80, 40001 (2007)

12. J.A. de Oliveira, C.P. Dettmann, D.R. da Costa, E.D. Leonel, Phys. Rev. E 87, 062904 (2013)

13. A.J. Lichtenberg, M.A. Lieberman, Regular and Chaotic Dynamics, Vol. 38 of Applied Mathematical Sciences (Springer Verlag, New York, 1992)

14. J.E. Howard, J. Humpherys, Physica D 80, 256 (1995)

15. B.V. Chirikov, Phys. Rep. 52, 263 (1979)

16. M.A. Lieberman, A.J. Lichtenberg, Phys. Rev. A 5, 1852 (1971)

17. J.K.L. da Silva, D.G. Ladeira, E.D. Leonel, P.V.E. McClintock, S.O. Kamphorst, Braz. J. Phys. 36, 700 (2006)

18. L.D. Pustylnikov, Trans. Moscow Math. Soc. 2, 1 (1978)

19. E.D. Leonel, P.V.E. McClintock, J. Phys. A 38, 823 (2005)

20. D.G. Ladeira, E.D. Leonel, Chaos 17, 013119 (2007)

21. E.D. Leonel, P.V.E. McClintock, J.K.L. da Silva, Phys. Rev. Lett. 93, 014101 (2004)

22. E.D. Leonel, Phys. Rev. Lett. 98, 114102 (2007)

23. G.A. Luna-Acosta, G. Orellana-Rivadeneyra, A. Mendoza-Galván, and C. Jung, Chaos, Solitons and Fractals, 12, 349 (2001)

24. E.D. Leonel, P.V. McClintock, Chaos 15, 033701 (2005)

25. E.D. Leonel, P.V.E. McClintock, Phys. Rev. E 70, 016214 (2004) 